\title{
Mathematical Modelling for the Diffusional Release of a Dispersed Solute from a Cylindrical Polymer Matrix into Finite External Volume
}

\author{
Thekkethil Shefeeq ${ }^{1}$, Naseem Ahmad ${ }^{2}$ \\ ${ }^{1}$ Centre for Interdisciplinary Research in Basic Sciences (CIRBSc), Jamia Millia Islamia, New Delhi, India \\ ${ }^{2}$ Department of Mathematics, Jamia Millia Islamia, New Delhi, India \\ Email: shefeeqjmi@gmail.com
}

Received October 18, 2011; revised November 24, 2011; accepted December 2, 2011

\begin{abstract}
An exact solution has been obtained for the release kinetics of a solute from a cylindrical non-erodible polymeric matrix into a finite external volume when the initial solute loading is greater than the solubility limit in the matrix. The moving boundary solution is derived based on the combination of variable method. The formulas of the moving boundary and the fractional solute release are given. The moving boundary and the fractional solute release profiles have been calculated at various solute loading levels and different external volumes. The obtained results show that as the external fluid volume increases, the fractional release at any time and the maximum fractional release increase. In addition, for a given external volume, as initial drug loading increases, the fractional release at any time decreases.
\end{abstract}

Keywords: Diffusional Release Kinetics; Exact Solution; Finite External Volume; Mathematical Modeling

\section{Introduction}

In recent years, the drug delivery field has experienced an exponential growth of interest in the role played by the carrier's geometry (i.e., shape and size) in its functions - drug loading and release, stability, toxicity and, ultimately, delivery performance. Research in the area of controlled drug delivery systems has become increasingly important due to their advantages in safety and efficacy. The purpose of these systems is to maintain desired drug concentration in the blood or in the tissue as long as possible.

Pharmaceutical controlled release systems, generally constructed from polymers, are defined as the systems which delivers a drug at a predetermined and constant rate for a specified period of time. Such systems exist in many forms, including injectable microspheres, specially designed tablets, implants and transdermal patches. Diffusion, degradation and dissolution are the most important mechanisms that control the drug release from these controlled release systems. Among these mechanisms, diffusion is the most important mechanism used to control the drug release from drug delivery systems [1].

Diffusional release of the drug is normally governed by Fick's first and second laws [2]. So far, several exact and approximate solutions have been developed to analyze the release kinetics of a dispersed solute from a po- lymeric matrix under a perfect sink condition. The diffusion-controlled mathematical model for dispersed drug delivery system in a planar sheet and a sphere was initiated by Higuchi $[3,4]$ with the assumption that diffusion is in pseudo-steady state. In the planar system, this assumption leads to a linear concentration profile of the drug in the diffusing region, which is between the diffusion front and the exposed surface of the device. Fick's first law is applied across this diffusion front, and this serves to determine the velocity and subsequently the position of the front as a function of time. However, this pseudo-stationary approximation is only appropriate when the initial solute loading $(A)$ is much greater than the solute solubility $\left(C_{s}\right)$ in the matrix. Since the diffusional front velocity should increase as solute loading decreases, the pseudo-steady state assumption is expected to be least applicable at low solute loadings. This drawback was removed by Paul and McSpadden [5] who achieved an exact solution for slabs under sink condition. Unfortunately, the exact solution involves a transcendental expression which is cumbersome for routine usage.

Lee [6] applied the refined integral method to develop an approximate explicit analytical solution which is simpler than McSpadden's solution but more accurate than Higuchi's results for small $A / C_{s}$ ratio where $A$ is initial solute loading per unit volume and $C_{s}$ is the solute solu- 
bility. Abdekhodaie and Cheng [7,8] obtained an exact solution for diffusional release of a dispersed solute from a spherical polymer matrix into both infinite and finite external medium by using a combination of variables technique. This technique allows the reduction of Fick's second law of diffusion into an ordinary differential equation.

$\mathrm{Wu}$ and Zhou $[9,10]$ developed a numerical solution for a finite external medium system with an additional diffusion boundary layer incorporated on the surface of the matrix. Again in 2005, they investigated dispersed drug release from two dimensional matrix tablets [11]. Recently Zhou et al. considered theoretical analysis of release kinetics of coated tablets containing constant and non-constant drug reservoirs [12]. Also Helbling et al. derived an explicit analytical solution for the case of controlled dispersed-drug release from planar non-erodible polymeric matrices based on Refined Integral Method [13]. Although many attempts have been made to analyze the release of a solute from a cylindrical polymeric matrix into both finite and infinite external medium the exact solution for the problem is absolutely scarce.

In the present study, the exact moving boundary solution for the release kinetics of a dispersed solute from a polymeric matrix of cylindrical geometry into a finite external volume is developed. The formulas of the moving boundary and the fractional solute release are given. Predicted fractional release profiles in cylindrical geometry are presented for various solute loading levels and different external volumes.

\section{The Mathematical Model for Diffusional Release}

The mathematical model for the diffusional release of a dispersed solute from a polymeric matrix with cylindrical geometry into finite volume has been considered with the following assumptions: 1) the diffusion coefficient is constant; 2) the system is cylindrical in shape with sealed ends so that the solute release from radius direction; 3 ) solute diffusion is assumed to be the rate controlling step rather than polymer swelling or drug dissolution; 4) the mass transfer resistance at the surface is negligible.

The cylindrical matrix in the dispersed solute system can be primarily divided into two regions: the surface region, $R<x<S$, in which all solute is dissolved, and the core region, $0<x<R$, in which the undissolved solute exists. Here, $S$ corresponds to the radius of the entire matrix, $R$ is the radius of the unextracted core and $r$ is the polar radial position. The two regions are separated by the diffusion front, $x=R$, which moves inward as time progress. The concentration in the unextrcted region $(r<$ $R)$ is constant and the concentration in the partially extracted region, $R<r<S$, is a function of both time and position variable $r$ and is determined by transient diffusion according to Fick's second law:

$$
\frac{\partial c}{\partial t}=\frac{D}{r} \frac{\partial}{\partial r}\left(r \frac{\partial c}{\partial r}\right)
$$

where $C$ is the concentration of solute in the polymeric matrix and $D$ is the diffusion coefficient. Assuming equilibrium between the surface and the external fluid at all times without any mass transfer resistance in the fluid, the initial and boundary conditions are:

$$
\begin{gathered}
C[R, t]=C_{s} \\
D \frac{\partial C}{\partial r}=\left(A-C_{s}\right) \frac{\mathrm{d} R}{\mathrm{~d} t} \text { at } r=R \\
C[S, t]=k C_{b} \\
R(0)=S
\end{gathered}
$$

where $C_{s}$ is the solubility limit of the solute in the matrix, $R(t)$ is the time dependent position of the moving diffusion front, $A$ is the initial solute loading per unit volume, $C_{b}(t)$ is the external bulk concentration at time $t$ and $k$ is the equilibrium distribution coefficient between the matrix and the external solution. Equation (4) states that the finite external medium concentration is related to the concentration at the surface $(r=S)$ by the partition coefficient $k$. It is more convenient to combine Equation (4) with a total mass balance at any time $t$ to obtain an alternative boundary condition:

$$
A\left\{1-\left(\frac{R}{S}\right)^{2}\right\}=C \lambda+\frac{2}{S} \int_{\mathrm{R}}^{S} C\left(\frac{r}{S}\right) \mathrm{d} r \text { at } r=S
$$

where $\lambda$ is the effective volume and is defined as,

$$
\lambda=\frac{V}{k \sigma S}
$$

where $V$ is the total external fluid volume and $\sigma$ is the surface area of the cylinder. The mathematical description of the problem then consists the governing Equation (1), and boundary and initial conditions Equation (2), Equation (3), Equation (5) and Equation (6).

Defining dimensionless variable as:

$$
\xi=\frac{r}{S} \quad \tau=\frac{D t}{S^{2}} \quad \Gamma=\frac{R}{S} \quad \theta=\frac{C}{C_{s}}
$$

Then the Equations (1), (2), (3), (6), and (5) can be reduced, respectively, to their dimensionless forms:

$$
\begin{gathered}
\frac{\partial \theta}{\partial \tau}=\frac{\partial^{2} \theta}{\partial \xi^{2}} \\
\theta[\Gamma(\tau), \tau]=1
\end{gathered}
$$




$$
\begin{gathered}
\frac{\partial \theta}{\partial \xi}=K \frac{\mathrm{d} \Gamma}{\mathrm{d} \tau} \text { at } \xi=\Gamma \\
\frac{A}{C_{s}}\left(1-\Gamma^{2}\right)=\theta \lambda+2 \int_{\Gamma}^{1} \xi \theta \mathrm{d} \xi \text { at } \xi=1 \\
\Gamma(0)=1
\end{gathered}
$$

where $K$ is defined as:

$$
K=\left(\frac{A}{C_{s}}-1\right)
$$

Now using the transformation:

$$
U=\frac{1-\xi}{\sqrt{\tau}}
$$

the dimensionless concentration $\theta$ becomes a total function of $U$ as follows:

$$
\theta(\xi, \tau)=\theta(U)
$$

The dimensionless diffusion equation, Equation (9), then becomes:

$$
\frac{\mathrm{d}^{2} \theta}{\mathrm{d} U^{2}}=-\left(\frac{U}{2}\right) \frac{\mathrm{d} \theta}{\mathrm{d} U}
$$

which yields on integrating:

$$
\theta=B_{1} \sqrt{\pi} \operatorname{erf}\left(\frac{U}{2}\right)+B_{2}
$$

where $B_{1}$ and $B_{2}$ are constants of integration.

By using boundary conditions (8) and (10), we get,

$$
\theta=1+B_{1} \sqrt{\pi}\left[\operatorname{erf}\left(\frac{1-\xi}{2 \sqrt{\tau}}-\right)-\operatorname{erf}\left(\frac{1-\Gamma}{2 \sqrt{\tau}}\right)\right]
$$

where $B_{1}$ is: (see Equation (20))

Using boundary condition Equation (11) gives:

$$
K \frac{\mathrm{d} \Gamma}{\mathrm{d} \tau}=\frac{-B_{1} \exp -\left(\frac{(1-\Gamma)^{2}}{4 \tau}\right)}{\sqrt{\tau}}
$$

The solution of this differential equation with initial condition Equation (13) should give the dimensionless moving front position as a function of time. But Equation (21) gives $\mathrm{d} \Gamma / \mathrm{d} \tau \rightarrow \infty$ at $\tau=0$, a solution for $\Gamma(\tau)$ cannot be obtained using the $\tau=0$ initial condition (Equation (13)). A value of $\Gamma$ at $\tau$ other than $\tau=0$ is needed as an initial condition for the numerical solu- tion of Equation (21). During a sufficiently short period of initial release, i.e. small $\tau$, the amount of released solute should be small enough that solute concentration in the external volume remains low and the diffusional driving force for release is not altered. Perfect sink condition can be assumed to apply during this initial period. Therefore at short times, release kinetics and the time profile of the moving diffusion front, $\Gamma(\tau)$, should both be independent of the size of the external volume. An exact solution for dispersed solute release from a planar matrix into a perfect sink has been developed by Paul and McSpadden [5]. Based on their solution, $\Gamma$ and $\tau$ can be related as follows:

$$
\sqrt{\pi} \frac{(1-\Gamma)}{2 \sqrt{\tau}} \exp \left[\frac{(1-\Gamma)^{2}}{4 \tau}\right] \operatorname{erf}\left[\frac{1-\Gamma}{2 \sqrt{\tau}}\right]=\frac{1}{K}
$$

By calculating $\Gamma$ for sufficintly small value of $\tau$ at any solute loading level from Equation (22) and using these values as an initial condition $\left(\Gamma_{0}, \tau_{0}\right), \Gamma$ verses $\tau$ profiles can be found by numerically solving Equation (21).

The cumulative amount of solute released, $M_{t}$, can be calculated by:

$$
M_{t}=C_{b} V=\frac{C(S, t) V}{k}=\frac{C_{s} \theta(1, \tau) V}{k}
$$

and the theoretical total amount of solute, or the amount releasable at infinite time, $M_{\infty}$, is :

$$
M_{\infty}=\sigma S A
$$

Combining Equation (19), Equation (23) and Equation (24), the resulting fractional release becomes:

$$
\frac{M_{t}}{M_{\infty}}=\frac{\lambda}{A / C_{s}}\left[1-B_{1} \sqrt{\pi} \operatorname{erf}\left(\frac{1-\Gamma}{2 \sqrt{\tau}}\right)\right]
$$

\section{Results and Discussion}

The fractional release, $\frac{M_{t}}{M_{\infty}}$, of a solute at time $t$ can be calculated from Equation (25) by substituting $\Gamma(\tau)$ profiles getting from the solution of Equation (21). But a solution for $\Gamma(\tau)$ cannot be obtained using the initial condition Equation (13). So we adopt the initial condition $\left(\Gamma_{0}, \tau_{0}\right)$ from Paul and McSpadden solution [5] for small $\tau$ as the curvature effect in the cylindrical geometry can be neglected at sufficiently small $\tau$. To use this approach, it

$$
B_{1}=\frac{K\left(1-\Gamma^{2}\right)-\lambda}{(2 \sqrt{\tau}-\lambda) \sqrt{\pi} \operatorname{erf}\left(\frac{1-\Gamma}{2 \sqrt{\tau}}\right)+2(1+\Gamma) \sqrt{\tau} \exp -\left(\frac{(1-\Gamma)^{2}}{4 \tau}\right)-4 \sqrt{\tau}}
$$


is necessary to determine if the solution for $\Gamma(\tau)$ is sensitive to the initial conditions selected. The duration of the initial period when perfect sink conditions pertain should depend on the effective volume of the external medium. Choosing a small effective volume, $\lambda=5$, when we expect great sensitivity, $\Gamma(\tau)$ solutions were obtained from Equation (21) using varying values of $\left(\Gamma_{0}, \tau_{0}\right)$ pairs from Equation (22) as the initial condition. The results for two

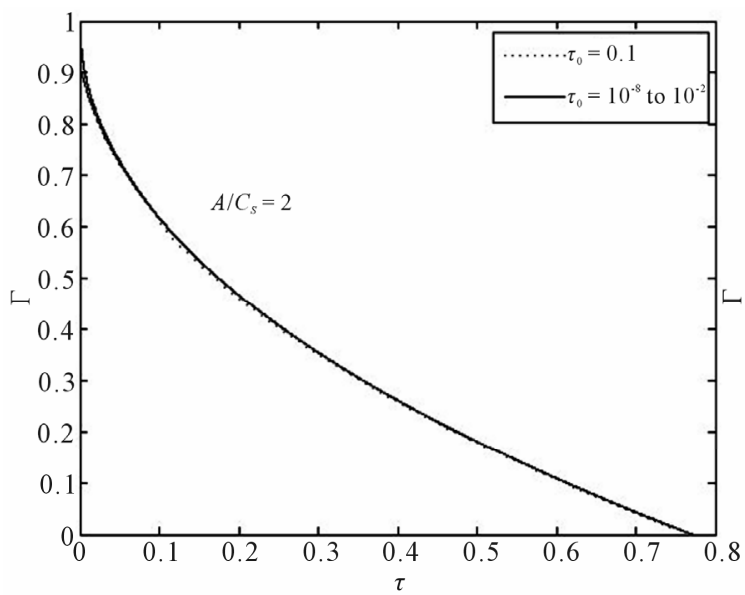

drug loadings, $A / C_{s}=2$ and 21, are shown in Figure 1. The results indicate that $\Gamma(\tau)$ profiles are insensitive to the initial conditions selected over a wide range of $\tau$, thus confirming the validity that ambient effects can be neglected at early times.

Fractional release profiles obtained using $\tau_{0}=10^{-4}$ for various solute loading levels and different values of $\lambda$ are shown in Figure 2. This figure shows that at a given

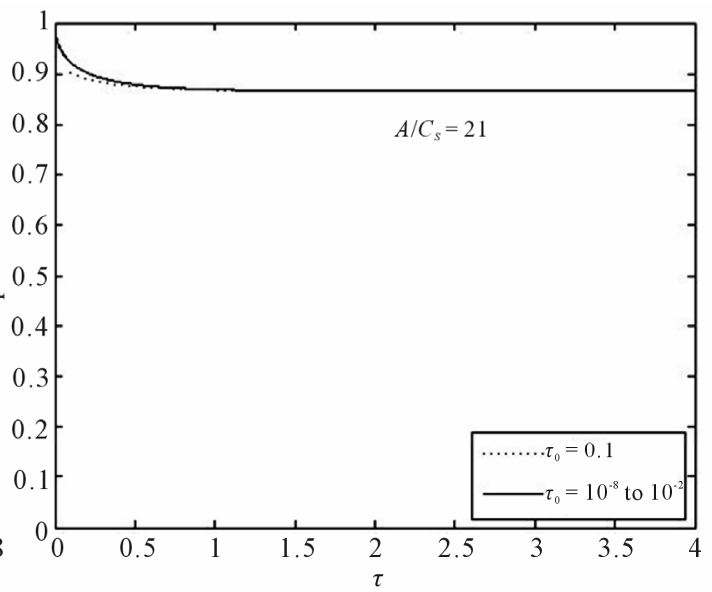

Figure 1. $\Gamma(\tau)$ for solute release from a cylindrical matrix into finite external volume $(\lambda=5)$ with initial condition $\Gamma_{0}$ at $\tau=\tau_{0}$, $\tau_{0}=10^{-8}$ to 0.1, obtained from Paul-McSpadden planar geometry solution [5], for two values of $A / C_{s}$.
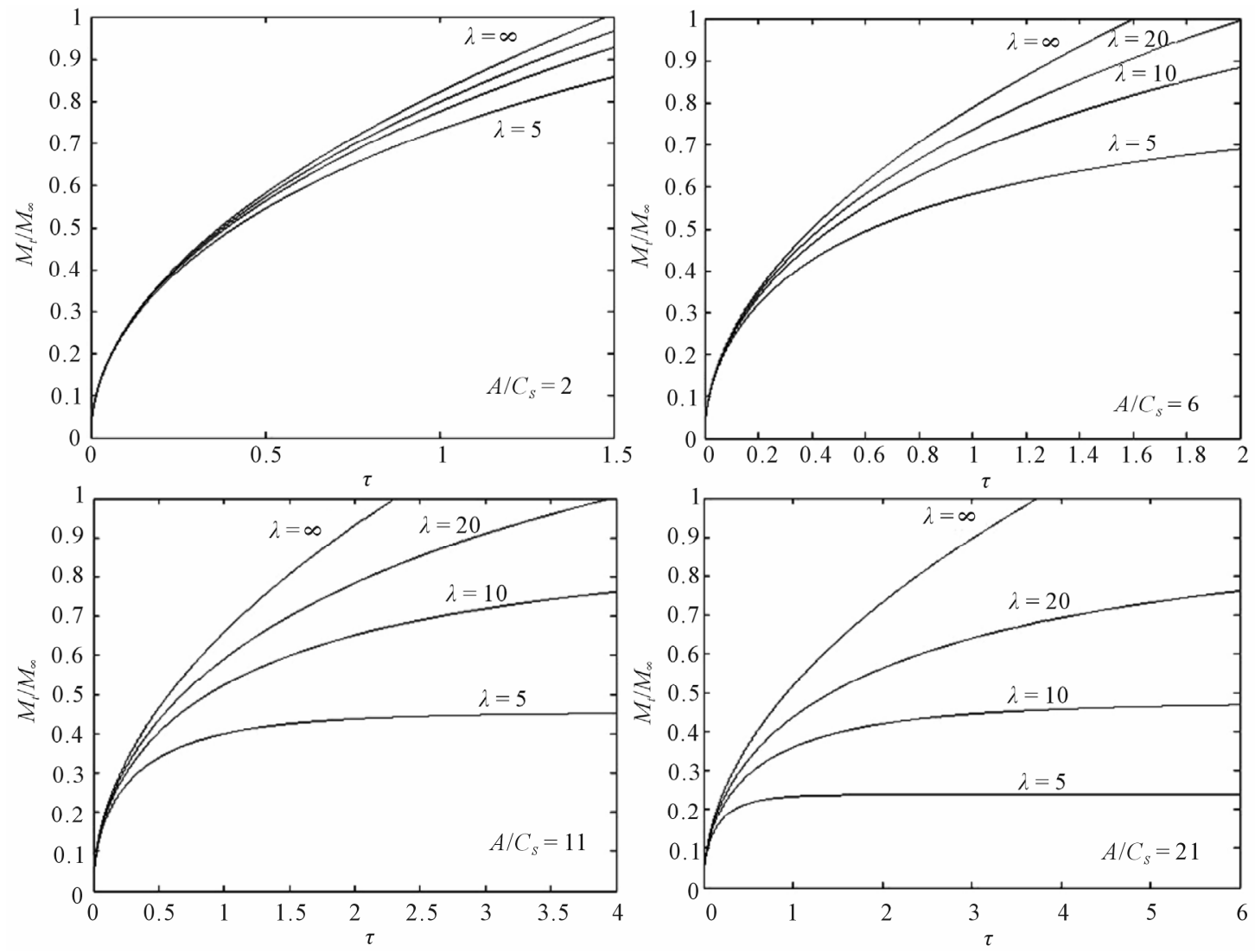

Figure 2. Fractional release for a dispersed solute in a cylindrical polymeric matrix at various solute loading levels and different external volumes. 
value of $A / C_{s}$, fractional release decreases as the external volume decreases. This result is consistent with the expectation that as external volume decreases, solute concentration build up in the external volume accelerates, and hence the rate of decrease in the diffusional driving force also accelerates.

\section{Conclusion}

An exact moving boundary solution has been presented and analyzed for the diffusional release of dispersed solute from a cylindrical matrix into a finite external medium. The moving diffusion front and the fractional solute release have been calculated with various drug loading levels and different external volumes. Figure 1 presents the variation of the position of the diffusion moving front with $A / C_{s}$ ratios. It can be noted that at lower drug loading the diffusional front should move inwards at a higher velocity. The comparison of this result with [8] shows that drug dissolution depends on initial loading and geometry of the drug release devices. Looking at Figure 2 we conclude that as the external fluid volume increases, the fractional release at any time increases. For any initial loading, the fractional solute release remains same up to a short time irrespective of different external volume. In addition, for a given external volume, as initial drug loading increases, the fractional release at any time decreases. These studies can help us to understand the mechanism of the controlled release systems.

\section{REFERENCES}

[1] J. Siepmann, H. Kranz, R. Bodmeier and N. A. Peppas, "HPMC-Matrices for Controlled Drug Delivery: A New Model Combining Diffusion, Swelling and Dissolution Mechanisms and Predicting the Release Kinetics," Pharmaceutical Research, Vol. 16, No. 11, 1999, pp. 17481756. doi:10.1023/A:1018914301328

[2] J. Crank, "Mathematics of Diffusion," 2nd Edition, Oxford University Press, Oxford, 1975.

[3] T. Higuchi, "Rate of Release of Medicaments from Ointment Bases Containing Drugs in Suspension," Journal of Pharmaceutical Sciences, Vol. 50, No. 10, 1961, pp. 874875. doi:10.1002/jps.2600501018
[4] T. Higuchi, "Mechanism of Sustained-Action Medication. Theoretical Analysis of Solid Drugs Dispersed in Solid Matrices," Journal of Pharmaceutical Sciences, Vol. 52, No. 12, 1963, pp. 1145-1149. doi:10.1002/jps.2600521210

[5] D. R. Paul and S. K. McSpadden, "Diffusional Release of a Solute from a Polymer Matrix," Journal of Membrane Science, Vol. 1, 1976, pp. 33-48. doi:10.1016/S0376-7388(00)82256-5

[6] P. I. Lee, "Diffusional Release of a Solute from a Polymeric Matrix. Approximate Analytical Solution," Journal of Membrane Science, Vol. 7, No. 3, 1980, pp. 255-275. doi:10.1016/S0376-7388(00)80472-X

[7] M. J. Abdekhodai and Y.-L. Cheng, "Diffusional Release of a Dispersed Solute from a Spherical Polymer Matrix," Journal of Membrane Science, Vol. 115, No. 2, 1996, pp. 171-178. doi:10.1016/0376-7388(96)00018-X

[8] M. J. Abdekhodai and Y.-L. Cheng, "Diffusional Release of a Dispersed Solute from a Dispersed solute from a Planar and Spherical Matrices into Finite External Volume," Journal of Controlled Release, Vol. 43, No. 2-3, 1997, pp. 175-182. doi:10.1016/S0168-3659(96)01482-4

[9] Y. Zhou and X. Y. Wu, "Theoretical Analysis of Dispersed-Drug Release from Planar Matrices with a Boundary Layer in a Finite Medium," Journal of Controlled Release, Vol. 84, No. 1-2, 2002, pp. 1-13. doi:10.1016/S0168-3659(02)00263-8

[10] Y. Zhou and X. Y. Wu, "Modeling and Analysis of Dispersed-Drug Release into a Finite Medium from Sphere Ensembles with a Boundary Layer," Journal of Controlled Release, Vol. 90, No.1, 2003, pp. 23-26. doi:10.1016/S0168-3659(03)00128-7

[11] Y. Zhou, J. S. Chu, T. Zhou and X. Y. Wu, "Modeling of Dispersed-Drug Release from Two-Dimensional Matrix Tablets," Biomaterials, Vol. 26, No. 8, 2005, pp. 945-952. doi:10.1016/j.biomaterials.2004.03.034

[12] Y. Zhou, J. S. Chu, J. X. Li and X. Y. Wu, "Theoretical Analysis of Release Kinetics of Coated Tablets Containing Constant and Non-Constant Drug Reservoirs," International Journal of Pharmaceutics, Vol. 385, No. 1-2, 2010, pp. 98-103. doi:10.1016/j.ijpharm.2009.10.039

[13] I. M. Helbling, J. C. D. Ibarra, J. A. Luna, M. I. Cabrera and R. J. A. Grau, "Modeling Dispersed-Drug Delivery from Planar Polymeric Systems: Optimizing Analytical Solutions," International Journal of Pharmaceutics, Vol. 400, No. 1-2, 2010, pp. 131-137. doi:10.1016/j.ijpharm.2010.08.043 